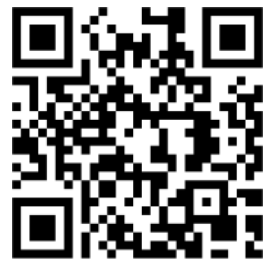

http://www.seer.ufms.br/index.ph $\mathrm{p} /$ pecibes/index

*Autor correspondente

Vanessa Ponsano Giglio.

E-mail do autor:

vanpg199@gmail.com

Palavras-chave:

Transtornos de deglutição. Acidente vascular cerebral. Tempo de internação.

Key-words: Deglutition disorders. Stroke. Length of stay.

\section{Disfagia pré-internação e sua relação com o tempo de permanência hospitalar}

Pre-hospitalization dysphagia and its relation with hospital length of stay

Giglio, Vanessa P. ${ }^{1}$, Oda, Adriana L. ${ }^{2}$, Daianese, Adriana C.L. ${ }^{3}$

${ }^{1}$ Fonoaudióloga, Hospital Universitário Maria Aparecida Pedrossian UFMS, Especialista em Fonoaudiologia Hospitalar e Disfagias Orofaringeas, Mestre em Saúde Coletiva pela UFMS.

${ }^{2}$ Fonoaudióloga, Coordenadora e Docente do Cefac e Neuroqualis, Doutorado em Neurologia pela Unifesp.

${ }^{3}$ Fonoaudióloga, Hospital Universitário Maria Aparecida Pedrossian UFMS, Especialista em Fonoaudiologia Hospitalar e Disfagias Orofaringeas pela Fac. Mozarteum de SP.

Resumo: O acidente vascular cerebral (AVC) apresenta elevada taxa de mortalidade e sequelas incapacitantes, configurando-se como importante problema de saúde pública. Dentre os fatores associados à incapacidade no AVC, destaca-se a disfagia orofaríngea neurogênica. Motivadas pelos relatos de dificuldades na alimentação vivenciadas por pacientes antes do acometimento neurológico, este estudo investigou se a queixa prévia de disfagia dos pacientes admitidos na Unidade de AVC do Hospital Universitário Maria Aparecida Pedrossian - UFMS impactou no aumento do tempo de internação quando comparados aos pacientes sem histórico e queixa de disfagia antes da internação. Trata-se de pesquisa de campo observacional, prospectiva, longitudinal, quantitativa e qualitativa. A casuística incluiu pacientes adultos de ambos os sexos, acima de 18 anos com ou sem queixa de disfagia, que responderam à triagem onde foram levantados dados indicativos da presença ou não da disfagia antes da internação. Após avaliação fonoaudiológica e estabelecimento da escala FOIS, os pacientes foram acompanhados até o momento da alta hospitalar. A Disfagia orofaríngea (DOF) foi identificada em $80 \%$ dos pacientes avaliados neste estudo. Foi significativa a associação entre não apresentar DOF e a ausência de queixa prévia, assim como não apresentar DOF e uma pontuação de NIHSS inicial menor que 10 pontos. Não houve associação entre o tempo de internação e a presença ou ausência de queixa prévia de DOF. Conclusão: Conclui-se que a investigação sobre a queixa prévia de disfagia nos pacientes acometidos por AVC pode fornecer dados norteadores para subsidiar o fonoaudiólogo durante a avaliação funcional da deglutição, porém, isoladamente, não demonstra relação estatisticamente significativa entre apresentar queixa prévia e o tempo de permanência hospitalar.

Abstract:Stroke has a high mortality rate and disabling after effects, representing an important public health problem. Among the factors associated with disability in stroke, neurogenic oropharyngeal dysphagia stands out. Motivated by the reports of difficulties in feeding experienced by patients before neurological impairment, this study sought to investigate whether the previous complaint of dysphagia of patients admitted to the stroke unit at Hospital Universitário Maria Aparecida Pedrossian - UFMS impacted the increase in hospital length of stay when compared to patients without a history and complaints of dysphagia before hospitalization. This regards an observational, prospective, longitudinal, quantitative and qualitative field research. The sample included adult patients of both sexes, over 18 years old with or without complaints of dysphagia, who responded to the screening where data were collected indicating the presence or not of dysphagia before hospitalization. After speech therapy evaluation and establishment of the FOIS scale, patients were followed up until the moment of hospital discharge. Oropharyngeal dysphagia (DOF) was identified in $80 \%$ of the patients evaluated in this study. There was a significant association between not having a DOF and the absence of a previous complaint, as well as not having a DOF and an initial NIHSS score of less than 10 points. There was no association between length of hospital stay and the presence or absence of a previous DOF complaint. Conclusion: It is concluded that the investigation of the previous complaint of dysphagia in patients affected by Stroke can provide guiding data to support the speech therapist during the functional evaluation of swallowing, however, in isolation, it does not demonstrate a statistically significant relationship between presenting a previous complaint and the length of hospital stay. 


\section{Introdução}

A atuação fonoaudiológica no ambiente hospitalar tem conquistado cada vez mais espaço voltado para a recuperação de pacientes acometidos por doenças crônicas com alterações na deglutição ${ }^{1}$. Sendo o fonoaudiólogo, o profissional capacitado a avaliar precocemente a deglutição e estabelecer a via de alimentação mais segura, estima-se que a presença deste profissional no ambiente hospitalar possa representar não só a redução de riscos associados à disfagia, mas também dos custos envolvidos numa internação prolongada.

A disfagia é definida como qualquer dificuldade de deglutição decorrente de processo agudo ou progressivo, que interfere no transporte do bolo desde a boca até o estômago ${ }^{2-}$ ${ }^{3}$ e que resulta na perda da funcionalidade e na independência para alimentar-se ${ }^{4}$. Não é considerada como uma doença, e sim, como um sintoma de uma doença ou a consequência de uma intervenção cirúrgica, podendo levar a graves complicações, como desidratação, desnutrição, aspiração de alimentos e, até mesmo à morte ${ }^{5}$. As complicações associadas à aspiração são comuns em pacientes com disfagia e podem determinar grave comprometimento pulmonar, aumentando a morbidade, o tempo de internação hospitalar, os custos envolvidos e o risco de morte nestes pacientes ${ }^{6}$.

Os distúrbios da deglutição foram reconhecidos pela OMS como uma incapacidade associada ao aumento da morbidade, mortalidade e custos com os cuidados necessários. Com o aumento das taxas de sobrevivência e o envelhecimento da população, os distúrbios da deglutição e as consequentes complicações pulmonares e nutricionais tornaram-se fator de extrema importância no contexto da saúde pública ${ }^{7}$.

Estudos demonstram que a disfagia tem um impacto significativo no tempo de internação hospitalar e é um indicador de mau prognóstico, sendo recomendado o reconhecimento precoce de disfagia e a intervenção no paciente hospitalizado para reduzir a morbidade e o tempo de internação hospitalar ${ }^{8}$. Para isso, o uso de uma triagem sistemática em disfagia pode resultar na diminuição significativa de casos de pneumonia aspirativa e na melhoria do estado geral do paciente ${ }^{9}$.

A disfagia pode ser decorrente de diversos fatores etiológicos, sendo a disfagia neurogênica pós Acidente Vascular Cerebral (AVC) bastante comum no ambiente hospitalar que atende a Linha de Cuidados em AVC na Rede de Atenção às Urgências e Emergências no SUS. A presença de disfagia orofaríngea é considerada uma das principais sequelas observadas após o acidente vascular encefálico, apresentando frequência variável entre 30 e $80 \%{ }^{10}$. Esta grande variação descrita na maioria dos estudos está provavelmente relacionada com a presença de amostras heterogêneas e com métodos distintos de investigação da deglutição orofaríngea ${ }^{11-12}$.

A vivência fonoaudiológica com estes pacientes tem demonstrado que, com frequência, o paciente acometido por disfagia pós-avc apresenta queixas relacionadas à alimentação previamente a internação e que, por motivos diversos, não são investigadas ou são subdiagnosticadas, mas representam um fator agravante nos pacientes com disfagia neurogênica, podendo ter relação com a gravidade do distúrbio e com o tempo de internação hospitalar. Considerando a complexidade de cuidados que o paciente diagnosticado com AVC requer, outros fatores também podem determinar o aumento no tempo de internação, tais como as comorbidades preexistentes, condições sociais, dificuldades no acesso a exames complementares necessários para o desfecho do tratamento e/ou agravamento do quadro neurológico.

Motivadas pelos relatos de dificuldades na alimentação vivenciadas por pacientes antes do acometimento neurológico, este estudo buscou investigar se a queixa prévia de disfagia dos pacientes admitidos na Unidade de AVC do Hospital Universitário Maria Aparecida Pedrossian - UFMS impactou no aumento do tempo de internação quando comparados aos pacientes sem histórico e queixa de disfagia antes da admissão na unidade hospitalar.

\section{Material e Métodos}

O presente estudo trata-se de uma pesquisa de campo, observacional, prospectiva, longitudinal, quantitativa e qualitativa que foi realizada durante dois meses consecutivos no Hospital Universitário Maria Aparecida Pedrossian, da Universidade Federal de Mato Grosso do Sul em Campo Grande/MS. Os sujeitos incluídos neste estudo foram pacientes adultos de ambos os sexos, acima de 18 anos com ou sem queixa de disfagia, em estado de alerta (respondendo a comandos simples) e em condições clínicas estáveis, admitidos na Unidade de Acidente Vascular Cerebral (UAVC) do Hospital Universitário Maria Aparecida Pedrossian. O estudo foi previamente autorizado pela Chefia da Unidade de Urgência e Emergência, setor responsável pela admissão dos pacientes da Unidade de AVC do Humap-UFMS, considerando a inexistência de custos orçamentários adicionais à instituição hospitalar.

Após a aprovação do Comitê de Ética em Pesquisa sob o parecer de número CAAE: 33637120.1.0000.5161, as pesquisadoras seguiram a rotina de atendimento fonoaudiológico adotada na instituição, com realização da avaliação fonoaudiológica em até 24 horas após admissão na Unidade de AVC e após a avaliação médica com o estabelecimento do diagnóstico neurológico. Foram excluídos deste estudo os sujeitos em estado de desorientação e/ou com baixo nível cognitivo (escala Glasgow abaixo 9) ${ }^{13}$, com instabilidade clínica, assim também como pacientes em uso de traqueostomia, ventilação mecânica, pacientes com intubação orotraqueal e pacientes em isolamento respiratório.

Para a realização desta pesquisa foram utilizados materiais básicos como formulários, prancheta, caneta, computador, copo, prato, colher e materiais específicos, como: abaixador de língua, lanterna, oxímetro, equipamentos de proteção individual, alimentos de diversas consistências e estetoscópio.

Após a apresentação do Termo de Consentimento Livre e Esclarecido, os pacientes e/ou acompanhantes responderam ao formulário de coleta de informações prévias, aqui denominado pelas pesquisadoras como "Triagem de risco para disfagia" onde foram investigadas as queixas relacionadas à presença de disfagia antes da admissão hospitalar. O questionário de triagem foi aplicado à beira do 
leito, abordando 12 questões abertas relacionadas à deglutição de alimentos de diversas consistências e sobre as possíveis consequências da disfagia, levantando dados indicativos da presença ou não da disfagia antes da internação.

Após a triagem, o paciente foi avaliado seguindo os itens do Protocolo de Avaliação Preliminar ${ }^{14}$ adaptado (PAP adaptado pelas pesquisadoras), que indicou a presença ou não da disfagia, possibilitando o estabelecendo do nível de ingesta oral de acordo com a escala FOIS - Functional Oral Intake Scale ${ }^{15-17}$.

Através da aplicação do Protocolo de Avaliação Preliminar adaptado (PAP adaptado) foram avaliados aspectos relacionados à respiração, fala, voz, estruturas orofaciais e cervicais. Foi realizada a avaliação quanto à mobilidade, tonicidade, sensibilidade e coordenação dos órgãos do sistema estomatognático, uma vez que as inadequações desta musculatura podem impactar negativamente nas funções de mastigação e deglutição. Toda a avaliação foi realizada no leito, estando o paciente sentado, alerta, com a cabeceira elevada a 45 graus ou mais, de acordo com a possibilidade de cada caso e ausência de contraindicação médica. Dados relacionados aos sinais vitais tais como frequência cardíaca (FC), Saturação de oxigênio (SpO2), assim também como nível de consciência, via de alimentação, comunicação, voz, respiração, dependência de oxigênio, condições estruturais da cavidade oral, dentição, uso de próteses dentárias, reflexos orais e manejo da deglutição de saliva foram considerados durante a avaliação dos pacientes.

A avaliação funcional da deglutição foi realizada com a oferta de alimentos de diferentes consistências por via oral, iniciando-se pela consistência semilíquida espessada, seguindo para consistência de líquidos finos e, por último, oferta de sólidos. Para a oferta de alimentos por via oral, foram considerados o desempenho do paciente em cada consistência, a segurança da oferta e as queixas referidas sobre saciedade ou fadiga. Os alimentos utilizados foram: suco diet de laranja espessado, água e bolacha de maisena, por se tratarem de alimentos disponíveis na rotina deste hospital, sem a necessidade de preparos especiais e/ou solicitações prévias ao setor de nutrição. Para as consistências de líquido fino (LF), foram realizadas 4 ofertas no copo, a saber: $2 \mathrm{ml}, 3$ $\mathrm{ml}, 5 \mathrm{ml}$ e gole livre. Para a consistência semilíquida espessada (SLE), foram usadas 3 medidas: colher "melada", colher rasa $(5 \mathrm{ml})$ e colher cheia $(10 \mathrm{ml})$. Para a consistência sólida foi utilizado bolacha com oferta livre.

A partir dos dados coletados na avaliação e, após determinação da presença de disfagia, os pacientes foram acompanhados pelo serviço de fonoaudiologia do HumapUFMS, de segunda a sexta-feira, no período matutino, seguindo a evolução clinica e fonoaudiológica até o momento da alta hospitalar, com o registro comparativo de evolução quanto à deglutição através da escala FOIS. A escala FOIS é um instrumento que possibilita avaliar a eficácia da fonoterapia na reabilitação da via oral e foi utilizada como marcador da progressão segura da dieta por via oral através do seu registro na admissão e na alta fonoaudiológica e/ou hospitalar pós-intervenção fonoaudiológica de acordo com os níveis de graduação de 01 a 07.
Para o seguimento fonoaudiológico foram empregadas estratégias facilitadoras que envolveram a estimulação tátil, térmica e gustativa, exercícios oromiofuncionais, manobras de deglutição, manobras de proteção das vias aéreas e ajustes posturais facilitadores para deglutição, além de orientações aos pacientes e cuidadores a respeito da modulação de consistência, controle de volume do alimento e do ritmo de oferta da alimentação.

Todos os dados coletados foram tabulados para posterior análise estatística, que possibilitou correlacionar as informações obtidas em cada fase de intervenção, desde a triagem, seguindo pela avaliação e no momento da alta hospitalar.

Os dados categóricos foram apresentados em frequência absoluta e relativa, os dados numéricos expressos em média \pm desvio padrão, dispostos em gráfico e tabela. As associações entre as variáveis foram calculadas pelo Teste Exato de Fisher (tabelas 2 x 2) ou pelo Teste Qui quadrado com correção de Bonferroni para análise de associação entre mais variáveis. A correlação entre o tempo de internação (dias) e a evolução da FOIS foi calculada pelo teste de correlação Linear de Spearman. Foi considerado um nível de significância de $5 \%$ e os testes realizados por meio do programa EPI INFOтм versão 7.2.2.6.

\section{Resultados}

O levantamento dos dados evidenciou que $100 \%$ dos pacientes avaliados receberam diagnóstico médico de Acidente Vascular Cerebral Isquêmico, dos quais 34\% foram submetidos à trombólise e $66 \%$ foram admitidos fora da janela terapêutica para inicio do tratamento com drogas fibrinolíticas ou com fatores de risco que determinaram contraindicação para este tipo de tratamento.

A idade dos participantes variou entre 30 e 96 anos, com média de 68,62 anos com desvio padrão de 13,43 anos, sendo $28(56 \%)$ do sexo masculino e $22(44 \%)$ do sexo feminino.

A Disfagia orofaríngea (DOF) foi identificada em $80 \%$ $(n=40)$ dos pacientes avaliados neste estudo. Foi significativa a associação entre não apresentar DOF e a ausência de queixa prévia $(\mathrm{p}=0,03)$, assim como não apresentar DOF e uma pontuação de NIHSS inicial menor que 10 pontos $(\mathrm{p}=0,007)$. A DOF não esteve associada à faixa etária $(\mathrm{p}=0,20)$, à pontuação de FOIS inicial ou final $(p=0,33$ e $p=1,00$, respectivamente) ou a pontuação de NIHSS final $(\mathrm{p}=0,47)$. (Tabela 1 na próxima página). 
Tabela 1. Distribuição de frequência dos 50 pacientes à associação de disfunção orofaríngea em relação às demais variáveis clínicas avaliadas. Campo Grande, 2020.

\begin{tabular}{|c|c|c|c|c|}
\hline \multirow[b]{2}{*}{ Variáveis } & \multirow{2}{*}{$\begin{array}{c}\text { Total } \\
(50)\end{array}$} & \multicolumn{2}{|c|}{ DOF } & \multirow{2}{*}{$\begin{array}{c}\text { Valor de } \\
\text { p }\end{array}$} \\
\hline & & $\begin{array}{l}\text { Não } \\
(10)\end{array}$ & $\begin{array}{l}\text { Sim } \\
(40)\end{array}$ & \\
\hline \multicolumn{5}{|l|}{ Faixa etária } \\
\hline 30 a 59 & $20,0(10)$ & $40,0(4)$ & $15,0(6)$ & \\
\hline 60 a 79 & $62,0(31)$ & $50,0(5)$ & $65,0(26)$ & 0,20 \\
\hline$\geq 80$ & $18,0(9)$ & $10,0(1)$ & $20,0(8)$ & \\
\hline \multicolumn{5}{|l|}{ Queixa prévia } \\
\hline Com queixa Sem & $42,0(21)$ & $10,0(1)$ & $50,0(20)$ & 0,03 \\
\hline queixa & $58,0(29)$ & $90,0(9)^{d}$ & $50,0(20)^{B}$ & \\
\hline \multicolumn{5}{|l|}{ OIS inicial } \\
\hline $1 \mathrm{a} 3$ & $12,0(6)$ & $0,0(0)$ & $15,0(6)$ & 033 \\
\hline$\geq 4$ & $88,0(44)$ & $100,0(10)$ & $85,0(34)$ &,$J$ \\
\hline \multicolumn{5}{|l|}{ FOIS final } \\
\hline $1 \mathrm{a} 3$ & $14,0(7)$ & $10,0(1)$ & $15,0(6)$ & 1.00 \\
\hline$\geq 4$ & $86,0(43)$ & $90,0(9)$ & $85,0(34)$ & \\
\hline \multicolumn{5}{|l|}{ NIHSS inicial } \\
\hline 0 a9 & $56,0(28)$ & $100,0(10)^{A}$ & $45,0(18)^{B}$ & \\
\hline 10 a19 & $36,0(18)$ & $0,0(0)$ & $45,0(18)$ & 0,00 \\
\hline$\geq 20$ & $8,0(4)$ & $0,0(0)$ & $10,0(4)$ & \\
\hline \multicolumn{5}{|l|}{ NIHSS final } \\
\hline 0 a9 & $84,0(42)$ & $100,0(10)$ & $80,0(32)$ & \\
\hline 10 a19 & $8,0(4)$ & $0,0(0)$ & $10,0(4) 2,5(1)$ & \\
\hline$\geq 20$. & $2,0(1)$ & $0,0(0)$ & & $0,47^{\circ}$ \\
\hline & $6,0(3)$ & $0,0(0)$ & $7,5(3)$ & \\
\hline
\end{tabular}

Nota: Dados apresentados como frequência relativa (frequência absoluta). ${ }^{a}$ teste do Qui quadrado com correção de Bonferroni; ${ }^{b}$ teste Exato de Fisher. Letras maiúsculas nas linhas apontam as diferenças entre os grupos.

As pontuações da FOIS inicial ou final não estiveram associadas à faixa etária dos participantes $(\mathrm{p}=0,39$ e $\mathrm{p}=0,30$, respectivamente), assim como não tiveram relação com a presença ou ausência de queixa prévia $(p=0,68$ e $p=1,00$, respectivamente) (Tabela 2 ).

Contudo, foi significativa a associação entre uma pontuação da FOIS $>4$ tanto inicial como final e um escore de NIHSS inicial inferior a 10 pontos $(\mathrm{p}=0,004 \mathrm{r} \mathrm{p}=0,02$; respectivamente). (Tabela 2)

Tabela 2 . Pontuações obtidas nas avaliações padronizadas FOIS em relação aos às demais variáveis clínicas avaliadas nos 50 pacientes. Campo Grande, 2020.

\begin{tabular}{|c|c|c|c|c|c|c|}
\hline \multirow[t]{2}{*}{ Variáveis } & \multicolumn{3}{|c|}{ FOIS inicial } & \multicolumn{3}{|c|}{ FOIS final } \\
\hline & 1 a 3 & valor de $p$ & & 1 a 3 & valor de $p>4$ & \\
\hline & (6) & (44) & & (7) & (43) & \\
\hline \multicolumn{7}{|l|}{ Faixa etária } \\
\hline $\begin{array}{l}30 \text { a } 59 \\
60 \text { a } 79\end{array}$ & $\begin{array}{r}0,0(0) \\
83,3(5)\end{array}$ & $\begin{array}{l}22,7(10) \\
59,1(26)\end{array}$ & $039^{a}$ & $\begin{array}{l}0,0(0) \\
85,7(6)\end{array}$ & $\begin{array}{l}23,3(10) \\
58,1(25)\end{array}$ & $0,30^{a}$ \\
\hline$\geq 80$ & $16,7(1)$ & $18,2(8)$ & & $14,3(1)$ & $18,6(8)$ & \\
\hline \multicolumn{7}{|l|}{ Queixa prévia } \\
\hline $\begin{array}{l}\text { Com queixa } \\
\text { Sem queixa }\end{array}$ & $\begin{array}{l}33,3(2) \\
66,7(4)\end{array}$ & $\begin{array}{r}43,2(19) \\
56,8(25)\end{array}$ & $1,00^{b}$ & $\begin{array}{l}28,6(2) \\
71,4(5)\end{array}$ & $\begin{array}{l}44,2(19) \\
55,8(24)\end{array}$ & $0,68^{b}$ \\
\hline \multicolumn{7}{|l|}{ NIHSS inicial } \\
\hline $0 \mathrm{~A} 9$ & $0,0(0)^{\mathrm{B}}$ & $63,4(28)^{A}$ & & & $\begin{array}{r}62,8 \\
(27)^{A}\end{array}$ & \\
\hline $\begin{array}{l}10 \text { a } 19 \\
\geq 20\end{array}$ & $\begin{array}{l}66,7(4) \\
33,3(2)\end{array}$ & $\begin{array}{r}31,8(14) \\
4,5(2)\end{array}$ & $0,004^{b}$ & $\begin{array}{c}14,3(1)^{\mathrm{B}} 57,1(4) \\
28,6(2)\end{array}$ & $\begin{array}{r}32,6(14) \\
4,7(2)\end{array}$ & $0,02^{b}$ \\
\hline
\end{tabular}

Obs: Dados apresentados como frequência relativa (frequência absoluta). Valores de FOIS apresentados em pontos. ${ }^{a}$ teste do Qui

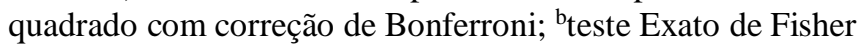

Não houve associação entre o tempo de internação e a presença ou ausência de queixa prévia de DOF $(\mathrm{p}=0,19)$ (Figura 1$)$.

Figura 1. Representação da associação entre o tempo de internação (dias) e a presença ou ausência de queixa prévia de DOF nos 50 pacientes. Campo Grande, 2020.

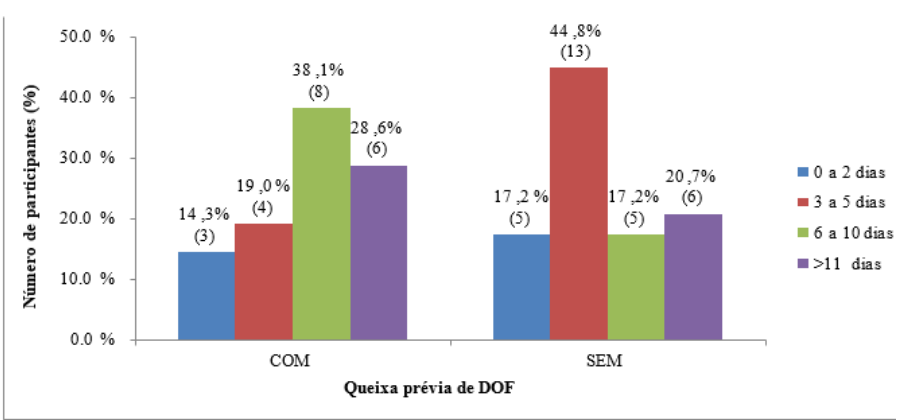

Nota: Teste qui quadrado $(\mathrm{p}=0,19)$

Apesar de apresentar uma correlação negativa entre o tempo de internação (dias) e a evolução da FOIS, ou seja, quanto menor o tempo de internação maior a evolução da FOIS, esta não foi estatisticamente significativa $(\mathrm{p}=0,21 ; \mathrm{r}=-0,18)$ (Figura 2$)$.

Figura 2. Representação da correlação entre o tempo de internação e a evolução da FOIS nos 50 pacientes. Campo Grande, 2020

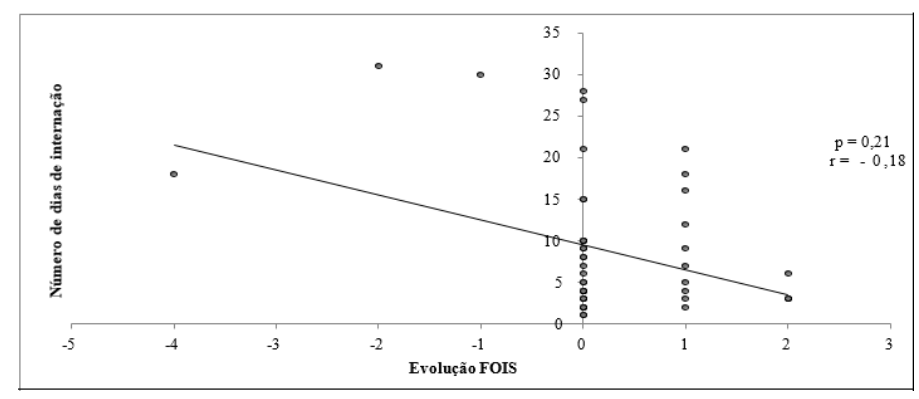

Teste de correlação Linear de Spearman

Figura 3. Representação da prevalência de DOF de acordo com as diferentes consistências nos 50 pacientes. Campo Grande, 2020

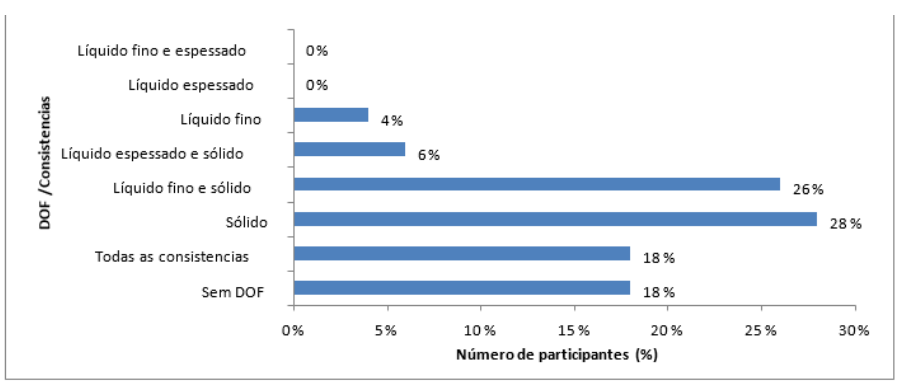

A alteração na força e no repouso das bochechas foi mais prevalente entre os pacientes com DOF $(\mathrm{p}=0,0007 \mathrm{e}$ 
$\mathrm{p}=0,03$; respectivamente). A alteração dos lábios em relação à força $(\mathrm{p}=0,01)$, mobilidade $(\mathrm{p}=0,0003)$ e repouso $(\mathrm{p}=0,008)$ também estiveram mais associadas aos pacientes com DOF, assim como em relação à força da língua $(p=0,03)$. Demais alterações estruturais não apresentaram associação entre a presença ou ausência de DOF ( $p>0,05)$, conforme detalhado na tabela 3 .

Tabela 3. Alterações identificadas na avaliação fonoaudiológica estrutural dos 50 pacientes. Campo Grande, 2020.

\begin{tabular}{|c|c|c|c|}
\hline \multirow[b]{2}{*}{ Avaliação fonoaudiológica estrutural } & \multicolumn{2}{|c|}{ DOF } & \multirow[b]{2}{*}{$\begin{array}{c}\text { Valor de } \\
\text { p }\end{array}$} \\
\hline & $\begin{array}{l}\text { Não } \\
(10)\end{array}$ & $\begin{array}{l}\text { Sim } \\
(\mathbf{4 0}\end{array}$ & \\
\hline \multicolumn{4}{|l|}{ Bochecha } \\
\hline Força alterada & $20,0(2)^{\mathrm{B}}$ & $80,0(32)^{A}$ & $0,0007^{b}$ \\
\hline Mobilidade alterada & $20,0(2)$ & $65,0(26)$ & $0,014^{b}$ \\
\hline Repouso alterado & $30,0(3)^{\mathrm{B}}$ & $70,0(28)^{A}$ & $0,03^{b}$ \\
\hline Sensibilidade alterada & $10,0(1)$ & $15,0(6)$ & $1,00^{\mathrm{b}}$ \\
\hline \multicolumn{4}{|l|}{ Lábios } \\
\hline Força alterada & $20,0(2)^{B}$ & $67,5(27)^{A}$ & $0,01^{\mathrm{b}}$ \\
\hline Mobilidade alterada & $20,0(2)^{\mathrm{B}}$ & $82,5(33)^{A}$ & $0,0003^{b}$ \\
\hline Repouso alterado & $0,0(0)^{\mathrm{B}}$ & $47,5(19)^{A}$ & $0,008^{b}$ \\
\hline Sensibilidade alterada & $20,0(2)$ & $17,5(7)$ & $1,00^{\mathrm{b}}$ \\
\hline \multicolumn{4}{|l|}{ Língua } \\
\hline Força alterada & $20,0(2)^{\mathrm{B}}$ & $62,5(25)^{A}$ & $0,03^{b}$ \\
\hline Mobilidade alterada & $20,0(2)$ & $50,0(20)$ & $0,15^{b}$ \\
\hline Repouso alterado & $0,0(0)$ & $0,0(0)$ & \\
\hline Sensibilidade alterada & $10,0(1)$ & $5,0(2)$ & $0,49^{b}$ \\
\hline Reflexo de GAG presente & & & \\
\hline $\begin{array}{l}\text { Reriexoc de GAG presente } \\
\text { Produço de saliva alterada }\end{array}$ & $\begin{array}{r}4,0(4) \\
0,0(0)\end{array}$ & $\begin{array}{r}0,0,0(2) \\
5,0(2)\end{array}$ & $1,00^{b}$ \\
\hline Alteração Qualidade Vocal & $10,0(1)$ & $20,0(8)$ & $0,67^{\mathrm{b}}$ \\
\hline Redução elevação laringea & $0,0(0)$ & $17,5(7)$ & $0,32^{\mathrm{b}}$ \\
\hline \multirow{2}{*}{\multicolumn{4}{|c|}{ Dentição }} \\
\hline Completa & & $5,0(2)$ & \\
\hline Incompleta & $60,0(6)$ & $67,5(27)$ & $0,61^{2}$ \\
\hline Ausente & $40,0(4)$ & $27,5(11)$ & \\
\hline Prótese dentária & $70,0(7)$ & $42,5(17)$ & $0,16^{b}$ \\
\hline Face Assimétrica & $40,0(4)$ & $75,0(30)$ & $0,06^{b}$ \\
\hline Ausência de comunicação & $0,0(6)$ & $15,0(6)$ & $0,33^{\mathrm{b}}$ \\
\hline
\end{tabular}

Obs: Dados apresentados como frequência relativa (frequência absoluta). ateste do Qui quadrado com correção de Bonferroni; bteste Exato de Fisher; (-) Teste estatístico não realizado por ausência de observações na categoria.

Durante a avaliação fonoaudiológica funcional foi identificada associação significativa entre a presença de DOF e a captação oral ineficiente $(\mathrm{p}=0,04)$, escape extra oral $(\mathrm{p}=0,01)$, trânsito oral lentificado $(\mathrm{p}<0,0001)$, mastigação ineficaz $(\mathrm{p}<0,0001)$ e presença resíduo oral $(\mathrm{p}=0,003)$. Não foram identificadas associações significativas entre demais itens avaliados e a DOF ( $p>0,05)$. Os valores estão detalhados na tabela 4.

Tabela 4. Alterações identificadas na avaliação fonoaudiológica funcional dos 50 pacientes. Campo Grande, 2020

\begin{tabular}{|c|c|c|c|}
\hline \multirow[b]{2}{*}{$\begin{array}{l}\text { Avaliação fonoaudiológica } \\
\text { funcional }\end{array}$} & \multicolumn{2}{|c|}{ DOF } & \multirow[b]{2}{*}{$\begin{array}{l}\text { Valor de } \\
\text { p }\end{array}$} \\
\hline & $\begin{array}{l}\text { Não } \\
(10)\end{array}$ & $\begin{array}{l}\text { Sim } \\
(40)\end{array}$ & \\
\hline Captação Oral ineficiente & $0,0(0)$ & $35,0(14)$ & $0,04^{*}$ \\
\hline Recusa alimentar & $0,0(0)$ & $5,0(2)$ & 1,00 \\
\hline Escape extra oral & $0,0(0)$ & $42,5(17)$ & $0,01^{*}$ \\
\hline Trânsito oral lentificado & $0,0(0)$ & $70,0(28)$ & $<0,0001^{*}$ \\
\hline Mastigação ineficaz & $0,0(0)$ & $95,0(38)$ & $<0,0001^{*}$ \\
\hline Presença Resíduo oral & $0,0(0)$ & $52,5(21)$ & $0,003^{*}$ \\
\hline Reduçã̃o da elevação laríngea & $0,0(0)$ & $5,0(2)$ & 1,00 \\
\hline \multicolumn{4}{|l|}{ Presença de Tosse } \\
\hline Antes & $0,0(0)$ & $0,0(0)$ & - \\
\hline Durante & $0,0(0)$ & $17,5(7)$ & 0,32 \\
\hline Após & $10,0(1)$ & $20,0(8)$ & 0,67 \\
\hline Pigarro & $20,0(2)$ & $22,5(9)$ & 1,00 \\
\hline Ausculta cervical + & $0,0(0)$ & $5,0(2)$ & 1,00 \\
\hline Qualidade vocal molhada & $0,0(0)$ & $2,5(1)$ & 1,00 \\
\hline Dispneia & $0,0(0)$ & $0,0(0)$ & - \\
\hline Queda na Saturação $\mathrm{O}_{2}$ & $0.0(0)$ & $0,0(0)$ & - \\
\hline Deglutição Incompleta & $0,0(0)$ & $0,0(0)$ & - \\
\hline Deglutição Múltipla & $0,0(0)$ & $30,0(12)$ & 0,09 \\
\hline Odinofagia & $0,0(0)$ & $0,0(0)$ & - \\
\hline
\end{tabular}

Obs: Dados apresentados como frequência relativa (frequência absoluta). ${ }^{a}$ teste do Qui quadrado com correção de Bonferroni; ${ }^{b}$ teste Exato de Fisher; (-) Teste estatístico não realizado por ausência de observações na categoria.

\section{Discussão}

Existem vários estudos que avaliaram as alterações na biomecânica da deglutição no indivíduo disfágico pós-AVE e o tempo de internação hospitalar, porém a associação dessas alterações e a presença de queixa de disfagia prévia à internação foram pouco relatadas.

$\mathrm{Na}$ presente pesquisa, do total de 50 pacientes diagnosticados com AVC, $100 \%$ apresentou AVC isquêmico confirmando a prevalência demonstrada em estudos anteriores com esta população ${ }^{18-20}$. Foram identificados $56 \%$ do sexo masculino e $44 \%$ do sexo feminino, não divergindo da literatura quanto aos dados epidemiológicos 4, 21-22. Os pacientes nessa população de AVC agudo incluíram 27 comorbidades gerais, porém, assim como outros estudos ${ }^{23}$, as complicações médicas agudas não foram incluídas em nossa análise devido à falta de dados suficientes sobre estas variáveis.

A literatura aponta que a disfagia está proeminentemente associada às idades mais avançadas. No período de 2005 a 2006, foram analisadas mais de 77 milhões de admissões hospitalares no National Hospital Discharge Survey (NHDS), das quais 271983 foram associadas à disfagia. Destes, observou-se que o grupo de pacientes com 75 anos ou mais apresentava uma taxa de disfagia de $0,73 \%$, o que corresponde a mais de duas vezes a média nacional para todas as demais faixas etárias no país onde a pesquisa foi desenvolvida ${ }^{8}$.

No presente estudo, porém, não foi possível relacionar a prevalência de disfagia nas faixas etárias comparadas. Apesar da maior prevalência de pacientes entre 60 a 79 anos apresentarem disfagia orofaríngea (DOF), esta faixa etária também foi prevalente no grupo de pacientes sem DOF. A diferença ligeiramente maior observada nesta faixa etária não se demonstrou estatisticamente significativa, podendo este fato ser justificado em decorrência do tamanho amostral da pesquisa. Como dos 50 pacientes, $62 \%$ está na faixa entre 60 e 79 anos, seria necessária uma prevalência de pacientes nas demais faixas etárias que nos possibilitasse a comparação entre elas para atingir uma diferença significativa.

Em concordância com estudos que relacionam a presença de disfagia pós-ave, foi identificada a presença de DOF em $80 \%$ dos pacientes avaliados neste estudo ${ }^{10,24-25}$.

No AVC agudo, a prevalência de disfagia foi relatada entre 28 e $65 \%$, uma variação que pode estar relacionada a diferenças na forma de avaliação da disfagia, na configuração e no tempo do teste empregado ${ }^{24}$. Outros estudos brasileiros também apontam para uma variação entre 48 e $91 \%$ na frequência de disfagia pós-AVC e correlacionam ao emprego de diferentes protocolos diagnósticos, além da realização da avaliação da deglutição em momentos e fases diversas, podendo ser aguda, subaguda ou crônica, evidenciando diferença nos resultados encontrados ${ }^{12,19,26}$.

A associação entre não apresentar DOF e a ausência da queixa prévia de disfagia $(\mathrm{p}=0,03)$ foi significativa e confirma a hipótese destas pesquisadoras quanto à 
importância de utilização de instrumentos que possam rastrear a presença de sintomas indicativos de disfagia previamente a internação e que muitas vezes são subconsiderados na anamnese e histórico do paciente. Houve uma relação entre não ter a DOF e não apresentar queixa prévia, ou seja, uma triagem que possibilite identificar a existência ou não da queixa prévia de DOF pode ser considerada como um indicativo simples, não oneroso e eficaz. Comprova-se, através dos resultados obtidos, que a maioria dos pacientes que não apresentou queixa prévia, não foi diagnosticado com DOF. Nesta linha de raciocínio, podemos afirmar que apresentar a queixa prévia não é indicativo de DOF, mas não ter tal queixa é indicativo de não apresentar tal disfunção.

Levando-se em consideração que a disfagia pode estar associada a fatores como queda na qualidade de vida, pneumonia aspirativa, desidratação, desnutrição e isolamento social, algumas diretrizes clínicas recomendam que o uso de instrumentos para a identificação precoce do risco de disfagia represente uma alternativa prática, de baixo custo e que acusa os casos em que uma avaliação mais detalhada torna-se necessária ${ }^{27-29}$.

A possibilidade de inserir uma triagem que busque rastrear as dificuldades vivenciadas pelo paciente durante a alimentação previamente a internação pós acometimento cerebrovascular parece ter efeito positivo e agregador de informações ao profissional que procederá à avaliação funcional da deglutição.

Para a avaliação clinica do paciente com acometimento neurológico, algumas escalas podem ser utilizadas para estabelecer o grau de comprometimento. O National Institutes of Health elaborou uma escala de gravidade para o AVC denominada de NIHSS (National Institutes of Health Stroke Scale) que possui evidências de confiabilidade clinicamente aceitáveis e boa aplicabilidade ${ }^{30}$. Cabe salientar, porém, que embora seja uma escala amplamente aceita e que aborde a avaliação clinica de diversos aspectos relacionados aos quadros neurológicos, não inclui a avaliação da deglutição ${ }^{15,31-32}$.

Pesquisas demonstram que pacientes com comprometimentos graves (NIHSS $\geq 16$ ) predizem uma alta probabilidade de morte ou severa incapacidade enquanto que um escore $\leq 6$ pode ser preditor de boa recuperação ${ }^{33}$. $\mathrm{Na}$ presente pesquisa houve relação entre os pacientes que não apresentaram DOF e uma pontuação de NIHSS inicial menor que 10 pontos $(\mathrm{p}=0,007)$. Apenas 8 pacientes apresentaram NIHSS inicial $\geq 16$, enquanto 19 pacientes obtiveram escore $\leq 6$ e 23 pacientes com escores entre 7 e 15 . Contudo, foi significativa a associação entre um escore de NIHSS inicial < 10 pontos e uma pontuação da FOIS > 4 tanto inicial como final. Nenhum paciente com NIHSS abaixo de 09 apresentou escala FOIS abaixo de 4, fato que exclui a necessidade de indicação de via alternativa. A determinação do nível de ingesta oral inicial estabelecida pela escala FOIS foi $\leq 3$ em apenas 6 pacientes com NIHSS acima de 10. Dos 44 pacientes que apresentaram FOIS inicial > 4, obtivemos 63,4\% dos sujeitos classificados com NIHSS até 9.

Não se pode afirmar no presente estudo que a FOIS se mostrou eficaz (ou adequada) para indicar a presença de DOF. Estatisticamente não houve uma associação entre uma pontuação ruim na escala FOIS e a presença de DOF. Ao contrário, todos os pacientes, independente de apresentarem ou não DOF, receberam pontuação acima de 4 na escala, correspondendo à possibilidade de liberação da alimentação por via oral, ainda que com modulações e/ou restrição de consistências. Dos 50 pacientes acompanhados, 33 não apresentaram evolução na escala FOIS inicial (estado agudo do AVC) e final (alta hospitalar). A evolução de nível na escala foi observada em 14 pacientes e 3 apresentaram complicações clinicas ao longo da internação com regressão do nível de pontuação. A presença de variáveis, tais como, a senescência, as condições odontológicas precárias e a presença de comorbidades podem estar associadas à manutenção do nível inicial da escala FOIS em muitos pacientes na alta hospitalar. Porém, cabe ressaltar que o momento da alta hospitalar nem sempre coincide com a alta fonoaudiológica, tampouco exclui a necessidade de continuidade da reabilitação.

Dentre todos os pacientes avaliados, $16 \%$ recebeu alta hospitalar em até dois dias, fato que pode estar associado aos baixos escores de NIHSS, que correspondem aos pacientes com menores riscos e sequelas decorrentes do $\mathrm{AVC}$ e às pontuações iguais ou acima de 4 na escala FOIS que possibilita a liberação da alimentação por via oral. No entanto, quando efetuamos a somatória dos pacientes com e sem DOF, observamos que o tempo de internação variou de 03 a 05 dias em $34 \%$, de 6 a 10 dias em $26 \%$ e acima de 11 dias em $24 \%$. Consideramos que o tempo de internação possa estar relacionado às demais variáveis, tais como a presença de comorbidades e intercorrências clínicas além da presença da DOF associada ou não à queixa prévia. Num estudo sobre a correlação da evolução da disfagia e a recuperação clínica no acidente vascular cerebral isquêmico, todos os cruzamentos das escalas de avaliação neurológica NIHSS e a Escala de Rankim modificada com as escalas FOIS e a Rosembek na fase aguda, após 30 e 90 dias do AVCi, obtiveram uma fraca correlação ${ }^{33}$. O estudo de coorte observacional retrospectivo realizado com 172 pacientes examinou 30 variáveis preditoras do tempo de permanência em um centro de reabilitação de AVC, destacando que, além da gravidade do AVC, outros fatores como a situação sócio-econômica e a medida de independência funcional na admissão podem estar correlacionados ao tempo de permanência ${ }^{34}$.

Este estudo não evidenciou uma associação direta entre o tempo de internação e presença ou ausência de queixa prévia de DOF. É possível analisarmos este dado de forma mais abrangente se considerarmos que o paciente em questão não foi internado por disfagia propriamente, sendo esta uma questão secundária ao motivo principal da internação. Em contrapartida, se a presença da queixa prévia nos pacientes pós AVC não teve associação com o tempo de permanência na unidade hospitalar, podemos afirmar, baseados em extensa literatura, que a presença da DOF pode acarretar não só o prolongamento do tempo de internação como também na ocupação de leitos, impedindo a rotatividade e gerando mais custos hospitalares. Um estudo de revisão e metanálise que abordou pesquisas realizadas nos Estados Unidos, Europa e Ásia observou um aumento no tempo de internação e nos custos com o grupo de paciente com disfagia orofaríngea. As despesas globais medidas via custo monetário aumentaram $40,36 \%$ em pacientes com disfagia orofaríngea em 
comparação com suas contrapartes não disfágicas ${ }^{35}$. Estudo de Patel et al. (2017) que avaliou impacto econômico das disfagia de origem orofaríngea e esofágica, revelou um aumento de $42 \%$ nos custos com a internação, apesar das diferenças na população, condição subjacente, ano ou país de origem dos estudos. Analogamente, a presença de disfagia orofaríngea acrescentou entre dois e oito dias extras ao tempo de internação hospitalar, independentemente da razão de internação ${ }^{36}$.

Muitas vezes as terminologias disfagia e broncoaspiração são empregadas equivocadamente de forma semelhante, visto a comprovada relação existente entre elas, à similaridade de circunstâncias em que estão envolvidas e ao impacto negativo que pode ser determinado quando o paciente disfágico apresenta também o risco de aspiração laringotraqueal de saliva e/ou alimentos. Cabe, porém, diferenciá-las de acordo com o que se avalia e como são interpretados os achados. A abordagem entre os sujeitos desta pesquisa considerou a disfagia em seu aspecto amplo, ressaltando as diversas fases da deglutição que garantem que a alimentação seja realizada de forma segura, com ganhos nutricionais e afetivos, levando-se em conta a segurança e o prazer alimentar. Constatamos que as alterações odontológicas podem impactar o processo de preparo oral durante a mastigação e na aceitação de determinadas consistências, destacando a consistência sólida que requer a presença de condições odontológicas satisfatórias e/ou presença de próteses dentárias que garantam que esta fase da deglutição seja desempenhada de forma efetiva 37. Encontramos um total de $50 \%$ dos pacientes avaliados com DOF para a consistência sólida associada com outras consistências e $28 \%$ com DOF apenas para sólidos, o que totaliza $78 \%$ dos pacientes com restrição a esta consistência podendo relacionar-se à dificuldade mastigatória e condições odontológicas precárias. Dados referentes à dentição apontaram que $95 \%$ dos pacientes com DOF apresentaram dentição incompleta ou ausente, sendo que destes apenas 42,5\% faziam uso de prótese dentária e $80 \%$ apresentou mastigação ineficaz durante a avaliação fonoaudiológica. Esta alteração justifica a dificuldade apresentada com a consistência sólida e níveis inalterados na escala FOIS mesmo com a intervenção fonoaudiológica, uma vez que modulações de consistência alimentar foram prescritas a fim de garantir a ingesta oral sem a possibilidade de evolução na escala. Com relação às demais consistências avaliadas, cabe salientar que a presença de sinais clínicos de disfagia com a consistência de líquido fino (48\%) esteve presente durante a sua oferta, enquanto que, a consistência de liquido espessado pareceu fornecer maior segurança durante a avaliação. Neste estudo, nenhum dos pacientes avaliados apresentou dificuldade com a consistência de liquido espessado isoladamente embora esta esteja presente quando associada à presença de disfagia com mais de uma consistência.

A utilização da escala FOIS como indicador de evolução realizada em um estudo com pacientes neurológicos e hospitalizados revelou que $73,5 \%$ dos 49 pacientes apresentaram avanço no nível da escala FOIS, indicando evolução com relação à funcionalidade da deglutição ${ }^{16}$. No entanto, similarmente aos resultados encontrados no presente estudo, Silvério et al. (2010) mencionam a pesquisa envolvendo 3 grupos diferentes de pacientes com TCE, demência e AVC, onde a maioria dos pós-AVC permaneceu no nível 5 da escala, com alimentação de múltiplas consistências, porém requerendo um preparo especial, até o final da intervenção fonoaudiológica hospitalar. As autoras observaram que a aplicação da escala FOIS foi capaz de demonstrar que com a intervenção fonoaudiológica há um avançar significativo de níveis da escala por parte dos pacientes com disfagia orofaríngea em âmbito hospitalar, no entanto, especificamente os pacientes pós-AVC permaneceram em níveis mais baixos da escala, necessitaram de maiores preparos e cuidados durante a alimentação até o término do atendimento fonoaudiológico hospitalar ${ }^{38}$.

Os dados observados na presente pesquisa quanto à manutenção do mesmo nível inicial e final na escala FOIS podem suscitar novos estudos que correlacionem as alterações próprias do envelhecimento e as condições odontológicas com impacto na mastigação e deglutição dos pacientes acometidos por acidente vascular cerebral.

De acordo com as Tabelas 3 e 4, os resultados obtidos demonstram, respectivamente, a prevalência de alterações quanto à postura, sensibilidade, força e mobilidade de OFAs (órgãos fonoarticulatórios) entre os pacientes com DOF, assim também como a maior prevalência de alterações funcionais, tais como, a captação oral ineficiente $(p=0,04)$, escape extra oral $(\mathrm{p}=0,01)$, trânsito oral lentificado $(\mathrm{p}<0,0001)$, mastigação ineficaz $(\mathrm{p}<0,0001)$ e a presença resíduo oral após a deglutição $(p=0,003)$.

$\mathrm{Na}$ amostra de sujeitos pesquisados por Carvalho et al. (2006), 52,9\% dos indivíduos apresentaram alteração na força labial que, por gerar um déficit de pressão intra-oral, causa desorganização da ejeção do bolo ${ }^{39}$. Além da diminuição da pressão intra-oral, a redução da força na oclusão labial implica em não retenção de líquido na cavidade oral e estase de alimentos no vestíbulo, configurando comprometimento das funções estomatognáticas ${ }^{40}$.

Considerando a fase oral com seus respectivos estágios de preparo, qualificação, organização e ejeção ${ }^{41}$, destacamos a existência de comprometimento desta fase nos achados desta pesquisa, conforme expressa a Tabela 4, destacando a captação ineficiente do alimento com consequente escape extra-oral (ou anterior) e a dificuldade no selamento labial completo pela não integridade morfofuncional das estruturas envolvidas na dinâmica da deglutição. Cabe ressaltar que as alterações encontradas pósave somam-se às alterações estruturais próprias do processo normal de envelhecimento, visto que esta pesquisa aborda a população de idosos.

Embora as alterações sensório-motoras relacionadas ao envelhecimento saudável possam contribuir para alterações voluntárias na ingestão alimentar, a presença de doença relacionada à idade, dentre elas o AVC, é o principal fator que contribui para a disfagia clinicamente significativa em idosos. Além das alterações motoras, os decréscimos relacionados à idade na umidade oral, no paladar e na acuidade do olfato podem contribuir para reduzir o desempenho da deglutição em idosos 42 .

As alterações relacionadas à força, mobilidade, sensibilidade e tônus das estruturas de lábio, língua e bochechas observadas nos resultados expressos na Tabela 3 
podem comprometer as funções de mastigação e deglutição, gerando alterações no mecanismo pressórico intra-oral e, consequentemente, nas demais fases da deglutição determinando a presença de DOF. Estes achados corroboram com os dados encontrados na literatura ${ }^{41,43-45}$.

A tosse é uma resposta reflexa do tronco cerebral que atua na proteção da via aérea contra a entrada de corpos estranhos. No presente estudo a presença da tosse foi observada em $37,5 \%$ dos pacientes com DOF, sendo mais prevalente durante $(17,5 \%)$ e após $(20 \%)$ a deglutição. A presença de tosse reflexa durante ou após a deglutição é considerada como um sinal de aspiração em decorrência de disfagia orofaríngea, podendo indicar a existência de sensibilidade na região laríngea e de habilidade para expectorar, ainda que não garanta o clareamento de via aérea 26

As alterações de deglutição podem ser avaliadas clinicamente ou através de uma avaliação instrumental buscando elucidar, de forma objetiva, dados que confirmem os achados clínicos para determinação do diagnóstico. Neste estudo, a avaliação estrutural e funcional realizada pelas fonoaudiólogas do serviço não teve o respaldo de avaliações objetivas, uma vez que estas não são oferecidas dentro da instituição hospitalar onde a pesquisa foi desenvolvida, fato considerado como uma limitação deste estudo, impossibilitando correlacionar os achados clínicos com a ocorrência de penetração ou aspiração laringotraqueal.

A presença da tosse foi observada em $37,5 \%$ dos pacientes com DOF, sendo mais prevalente durante $(17,5 \%)$ e após (20\%) a deglutição. Demais alterações encontradas nesta pesquisa, tais como, pigarro, ausculta cervical positiva, qualidade vocal molhada, dispneia, queda de $\mathrm{SpO} 2$, deglutições incompletas, deglutições múltiplas e odinofagia, embora comumente relatadas na literatura 26,32,39,46-48 compondo os quadros de disfagia, não despontaram relevância estatística neste estudo quando comparadas ao grupo de pacientes sem DOF.

\section{Considerações finais}

Os resultados indicam que a investigação sobre a queixa prévia de disfagia nos pacientes acometidos por Acidente Vascular Cerebral pode fornecer dados norteadores para subsidiar o fonoaudiólogo durante a avaliação funcional da deglutição, porém, isoladamente, não demonstram relação estatisticamente significativa entre apresentar queixa prévia e o tempo de permanência hospitalar.

Comparados a outras pesquisas internacionais, os achados deste estudo trazem importante contribuição para o aprofundamento do tema investigado, propondo a abordagem de novas pesquisas nessa temática, buscando comprovar a importância da investigação pormenorizada sobre a história pregressa à hospitalização dos pacientes disfágicos e os diferentes desfechos clínicos durante o período de internação.

\section{Referências}

1 - Faria, KCF; Pessoa, ACN; Araújo, LI; Paiva, MLF. Perfil do paciente atendido pela fonoaudiologia na unidade de urgência e emergência de um hospital universitário. Audiol., Commun. Res. v.18 n.4 São Paulo Oct./Dec. 2013

2- Macedo-Filho ED; Gomes GF ; Furkim, AM. Manual de cuidados do paciente com Disfagia. São Paulo: Ed Lovise; 2000. p.29.

3- Furquim, AM; Barata,L.; Duarte,ST; Nascimento Jr. Gerenciamento fonoaudiologico da disfagia no paciente critico na Unidade de terapia Intensiva. In: Furkim, AM; Rodrigues, KA. Disfagias nas Unidades de terapia Intensiva. $1^{a}$ ed. São Paulo: Ed.Roca, 2014, p.111.

4- Mourao, AM et al . Frequência e fatores associado à disfagia após acidente vascular cerebral. CoDAS, São Paulo , v. 28, n. 1, p. 66-70, Feb. 2016 Disponível em https://doi.org/10.1590/2317-1782/20162015072.

5- Sassi, FC; Medeiros, GC; Zambon, LC; Zilberstein, B; Andrade, CRF. Avaliação e classificação da disfagia pós-extubação em pacientes críticos. Rev Col Bras Cir. 45(3):e1687, 2018.

6-Toufen Junior, C; Camargo, FP; Carvalho, CRR. Pneumonia aspirativa associada a alterações da deglutição: relato de caso. Rev. bras. ter. intensiva, São Paulo, v. 19, n. 1, p. 118-122, Mar. 2007.

7- Rommel, N; Hamdy S. Oropharyngeal dysphagia: manifestations and diagnosis. Nat Rev Gastroenterol Hepatol. 2016;13(1):49- 59. doi:10.1038/nrgastro.2015.199.

8- Altman, KW, Yu G, Schaefer SD. Consequence of dysphagia in the hospitalized patient: impact on prognosis and hospital resources. Arch Otolaryngol Head Neck Surg. 2010;8:784-9.

9- Dennis, MS, Lewis SC, Warlow C; FOOD Trial Collaboration. Effect of timing and method of enteral tube feeding for dysphagic stroke patients (FOOD): a multicentre randomized controlled trial. Lancet. 2005;365(9461):76472 .

10 - Martino,R; Foley N; Bhogal S; Diamant N; Speechley M; Teasell R. Dysphagia after stroke: incidence, diagnosis, and pulmonary complications. Stroke. 2005;36(12):27562763. doi:10.1161/01.STR.0000190056.76543.eb.

11- Mann, G, Hankey GJ, Cameron D. Swallowing disorders following acute stroke:prevalence and diagnostic accuracy. Cerebrovasc Dis. 2000;10(5):380-6.

12- Schelp, AO et al . Incidência de disfagia orofaríngea após acidente vascular encefálico em hospital público de referência. Arq. Neuro-Psiquiatr., São Paulo, v. 62, n. 2b, p. 503-506, Junho 2004. Disponível em. https://doi.org/10.1590/S0004-282X2004000300023.

13- Furmann, N.; Costa, FM. Critérios clínicos utilizados por profissionais para liberação de dieta via oral em pacientes adultos hospitalizados. Rev. CEFAC [online]. 2015, vol.17, n.4, pp.1278-1287. ISSN 1516-1846. http://dx.doi.org/10.1590/1982-0216201517413614. 
14-Mangilli, LD, Moraes DP, Medeiros GC. Protocolo de avaliação fonoaudiológica preliminar. In: Andrade CRF, Limongi SCO (Org). Disfagia: prática baseada em evidências. São Paulo: Sarvier; 2012. p. 45-61.

15 - Crary, MA, Mann GD, Groher ME. Initial psychometric assessment of a functional oral intake scale for dysphagia in stroke patients. Arch Phys Med Rehabil. 2005;86(8):1516-1520. doi:10.1016/j.apmr.2004.11.049

16 - Furkim,AM; Sacco,ABF. Eficácia da fonoterapia em disfagia neurogênica usando a escala funcional de ingestão por via oral (FOIS) como marcador. Rev CEFAC, São Paulo, v.10, n.4, 503-512, out-dez, 2008.

17- Silva, RG, Jorge AG, Peres FM, Cola PC, Gatto AR, Spadotto AA. Protocolo de eficácia terapêutica em disfagia orofaríngea neurogênica (Procedon). Rev. CEFAC. 2010 Jan-Fev; 12(1):75-81.

18- Almeida, EO; Faleiros BE; Martins C; Lemos SMA; Teixeira AL. Características clínicodemográficas dos acidentes vasculares encefálicos de pacientes atendidos no Hospital Público Regional de Betim, MG. Rev Med Minas Gerais. 2011;21(4):384-9.

19- Mourão, AM et al. Evolução da deglutição no pósAVC agudo:estudo descritivo. Rev. CEFAC, São Paulo , v. 18, n. 2, p. 417-425, abr. 2016. Disponível em http://dx.doi.org/10.1590/1982-0216201618212315.

20- Merel S Ekker, Esther M Boot, Aneesh B Singhal, Kay Sin Tan, Stephanie Debette, Anil M Tuladhar, Frank-Erik de Leeuw. Epidemiology, aetiology, and management of ischaemic stroke in young adults. The Lancet Neurology, Volume 17, Issue 9, 2018, Disponível em: doi.org/10.1016/S1474-4422(18)30233-3.

21- Falcão, IV; Carvalho EMF de; Barreto KML; Lessa FJD; Leite VMM. Acidente vascular precoce: implicações para adultos em idade produtiva atendidos pelo Sistema Único de Saúde. Rev. Bras. Saúde Matern. Infant. 2004;4(1):95-102.

22- Locatelli, MC; Furlaneto, AF; Cattaneo,TN. Perfil epidemiológico dos pacientes com acidente vascular cerebral isquêmico atendidos em um hospital . Rev Soc Bras Clin Med. 2017 jul-set;15(3):150-4.

23- Luvizutto GJ, Gameiro MOO, Schelp AO, Braga GP, Ribeiro PW, Bazan R. Characterization of patients treated by rehabilitation service after establishing of an acute stroke unit in a Brazilian hospital. J Phys Ther Sci. 2015;27(8):2533-6. Disponivel em: https://doi.org/10.1589/jpts.27.2533.

24- Cohen, DL, Roffe C, Beavan J, et al. Post-stroke dysphagia: A review and design considerations for future trials.Int $\mathbf{J}$ Stroke.2016;11(4):399-411. doi:10.1177/1747493016639057.

25 - Jones CA, Colletti CM, Ding MC. Post-stroke Dysphagia: Recent Insights and Unanswered Questions. Curr Neurol Neurosci Rep. 2020 Nov . Disponível em doi: 10.1007/s11910-020-01081-z.

26- Padovani, AR et al . Protocolo fonoaudiológico de avaliação do risco para disfagia (PARD). Rev. soc. bras. fonoaudiol., São Paulo, v. 12, n. 3, p. 199-205, Set. 2007.
Disponível em 80342007000300007

27- Andrade, PA et al . Importância do rastreamento de disfagia e da avaliação nutricional em pacientes hospitalizados. Einstein (São Paulo), São Paulo , v. 16, n. 2, eAO4189, 2018. Epub June 07, 2018. Disponível em : https://doi.org/10.1590/s1679-45082018ao4189.

28- Gonçalves, MI, Remaili CB, Behlau M. Cross-cultural adaptation of the Brazilian version of the Eating Assessment ToolEAT-10. Codas. 2013;25(6):601-4.

29- Boccardi, V, Ruggiero C, Patriti A, Marano L. Diagnostic Assessment and Management of Dysphagia in Patients with Alzheimer's Disease. $J$ Alzheimers Dis. 2016;50(4):947-955. doi:10.3233/JAD-150931.

30- Caneda, M.A.G.de et al . Confiabilidade de escalas de comprometimento neurológico em pacientes com acidente vascular cerebral. Arq. Neuro - Psiquiatr., São Paulo,v.64, n.3a, p.690697, Set. 2006. Disponível em http://dx.doi.org/10.1590/S0004-282X2006000400034.

31- Okubo, PCMI. Detecção de disfagia na fase aguda do acidente vascular cerebral isquêmico: proposição de conduta baseada na caracterização dos fatores de risco. Tese (Doutorado em Neurologia) - Fac. de Medicina de Ribeirão Preto, Universidade de São Paulo, 2008. Doi: 10.11606/T.17.2008.tde-11092008-145314.

32- Itaquy, RB et al . Disfagia e acidente vascular cerebral: relação entre o grau de severidade e o nível de comprometimento neurológico. J. Soc. Bras. Fonoaudiol., São Paulo, v. 23 , n. 4, p. 385-389, Dec. 2011.Disponível em: https://doi.org/10.1590/S217964912011000400016

33- Nunes, MCA. Correlação da evolução da disfagia $e$ a recuperação clínica no Acidente Vascular Cerebral isquêmico.Tese (Doutorado Programa de Medicina Interna e Ciências da Saúde) - Universidade Federal do Paraná. Curitiba, 2016. Disponível:https://www.prppg.ufpr.br/siga/visitante/traba lhoConclusaoWS ?idpessoal=17830\&idprograma $=4$ 0001016012P1\&anobase $=2016 \&$ idtc $=13$.

34 - García-Rudolph A, Cegarra B, Opisso E, Tormos JM, Bernabeu M, Saurí J. Predicting length of stay in patients admitted to stroke rehabilitation with severe and moderate levels of functional impairments. Medicine (Baltimore). 2020 Oct 23;99(43):e22423. doi: 10.1097/MD.0000000000022423. PMID: 33120737; PMCID: PMC7581132.

35- Attrill, S., White, S., Murray, J. et al. Impact of oropharyngeal dysphagia on healthcare cost and length of stay in hospital: a systematic review. BMC Health Serv Res 18, 594 (2018). https://doi.org/10.1186/s12913-018-3376-3

36- Patel, DA, Krishnaswami S, Steger E, Conover E, Vaezi MF, Ciucci MR, Francis DO. Economic and survival burden of dysphagia among inpatients in the United States. Dis Esophagus. 2017.

37- Giglio, VP. "Decifra-me ou Devoro-te": Dificuldades de alimentação dos idosos e seus enigmas. Dissertação 
(Mestrado em Saúde Coletiva) - UFMS. 2003. Disponivel em: https://repositorio.ufms.br:8443/jspui/handle/123456789 1291.

38- Silvério CC, Hernandez AM, Gonçalves MIR. Ingesta oral do paciente hospitalizado com disfagia orofaríngea neurogênica. Revista CEFAC. 2010; 12(6): 964-970. Disponível:http://www.scielo.br/scielo.php?script=sci_a rttext\&pid=S1516-18462010000600007\&lng=en. Epub Aug 20,2010. http://dx.doi.org/10.1590/S151618462010005000090 .

39- Carvalho, YSV, Xerez DR, Araujo, AQC. Identificação de broncoaspiração por disfagia orofaríngea em pacientes com pneumonia comunitária. Acta Fisiátrica. 2006; 13(2):59-62. Disponivel em: http://www.actafisiatrica.org.br/detalhe_artigo.asp?id=2 21.

40- Tessitore, A; Pfelsticker, LN; Paschoal, JR. Aspectos neurofisiológicos da musculatura facial permitindo reabilitação na paralisia facial. Rev.CEFAC, São Paulo, v. 10,n.1, p. 68-75, março de 2008. Disponível em :http://www.scielo.br/scielo.php?script=sci_arttext\&pid $=\mathrm{S} 151618462008000100010 \& \operatorname{lng}=\mathrm{en} \& \mathrm{nrm}=\mathrm{iso}>$.

41- Yamada, EK et al . A influência das fases oral e faríngea na dinâmica da deglutição. Arq. Gastroenterol., São Paulo, v.41, n.1, p.18-23, Mar.2004. Disponível:http://www.scielo.br/scielo.php?script=sci_a rttext\&pid=S000428032004000100004\&lng=en\&nrm=i so.

42 - Sura, L., Madhavan, A., Carnaby, G., \& Crary, M. A. (2012). Dysphagia in the elderly: management and nutritional considerations. Clinical interventions in aging, 7, 287298. https://doi.org/10.2147/CIA.S23404.

43 -Silva, Lúcia Marilac da. Disfagia orofaríngea pós-acidente vascular encefálico no idoso. Rev. bras. geriatr. gerontol, Rio de Janeiro, v.9, n.2, p.93-106, agosto de 2006.Disponível:http://www.scielo.br/scielo.php?script =sci_arttext\&pid=S180998232006000200093\&lng=en $\&$ nrm $=$ iso $>$.

44- Warabi T; Ito T; Kato M; Takei H; Kobayashi N; Chiba S. Effects of stroke-induced damage to swallow-related areas in the brain on swallowing mechanics of elderly patients. Geriatr Gerontol Int. 2008;8(4):234-242. Disponível em: doi:10.1111/j.1447-0594.2008.00473.x.

45- Queiroz, MA dos Santos de; Haguette, RCB; Haguette, EF. Achados da videoendoscopia da deglutição em adultos com disfagia orofaríngea neurogênica. Revista da SBFa., São Paulo, v.14, n.4, p.454-462, 2009. Disponível: http://www.scielo.br/scielo.php?script=sci_arttext\&pid= S151680342009000400005\&lng=en\&nrm=iso.

46- Buchholz, DW. Dysphagia associated with neurological disorders. Acta Otorhinolaryngol Belg. 1994;48(2):143155.

47- Brasil. Ministério da Saúde. Secretaria de Atenção à Saúde. Departamento de Ações Programáticas Estratégicas. Diretrizes de atenção à reabilitação da pessoa
AVC.Brasília/DF,2013.Disponivel:http://bvsms.saude.g ov.br/bvs/publicacoes/diretrizes_atencao_reabilitacao_a cidente_vascular_cerebral.pdf.

48- Jacques, A, \& Cardoso, MC. de A F (2011). Acidente Vascular Cerebral e sequelas fonoaudiológicas. Rev Neurociências,19(2),229-236. Disponivel em: https://doi.org/10.34024/rnc.2011.v19.8371. 